\title{
Coherent excitation of a nonlinear microcavity
}

J. Oden

\section{S. Trebaol}

\section{P. Delaye}

\section{N. Dubreuil \\ nicolas.dubreuil@institutoptique.fr}

\author{
Laboratoire Charles Fabry, Institut d'Optique, CNRS, Univ Paris Sud, 2 Avenue Augustin Fresnel, \\ 91127 PALAISEAU cedex, FRANCE \\ Laboratoire Charles Fabry, Institut d'Optique, CNRS, Univ Paris Sud, 2 Avenue Augustin Fresnel, \\ 91127 PALAISEAU cedex, FRANCE \\ Laboratoire Charles Fabry, Institut d'Optique, CNRS, Univ Paris Sud, 2 Avenue Augustin Fresnel, \\ 91127 PALAISEAU cedex, FRANCE \\ Laboratoire Charles Fabry, Institut d'Optique, CNRS, Univ Paris Sud, 2 Avenue Augustin Fresnel, \\ 91127 PALAISEAU cedex, FRANCE
}

Coherent excitation of a nonlinear semiconductor microcavity is theoretically reported. It intends to counterbalance the frequency drift of the cavity resonance driven by the nonlinear refractive effects, which causes a limitation in the energy coupling efficiency of an input pulse into the cavity resonance. We show that exciting such a nonlinear microcavity with tailored chirped pulses allows to maintain the benefit of light localization and to further enhance light-matter interactions, opening the way to the realization of highly efficient nonlinear devices. [DOI: http://dx.doi.org/10.2971/jeos.2013.13046]

Keywords: Optical microcavities, nonlinear optics, coherent control, photonic crystal microcavities

\section{INTRODUCTION}

Photonic crystal microcavities with high-quality factor and very small mode volume allow a strong enhancement of lightmatter and nonlinear interactions [1]. Among the various key components for photonic signal processing, realizations of alloptical switches based on nonlinear photonic crystal microcavities have been reported in Silicon [2], InP [3] (containing quantum wells), GaAs [4], InGaAs [5], and GaInP [6]. In such a device, a control pulse induces a refractive index change of the nonlinear material, which in turns shifts the resonance frequency and modifies the transmission of the cavity. The refractive index variation is achieved through the plasma effect of carriers generated by either one-photon [3] or twophoton absorption [2, 4, 5], and ultimately by means of the optical Kerr effect [6]. Whereas the nonlinear refractive index change induced by the pulse front edge detunes the cavity resonance, it also contributes to a decrease of the coupling efficiency for the rest of the pulse. It means that only one part of the pulse energy is effective to perform the optical switching operation. Despite this limitation in the energy coupling efficiency, the mismatch between the time varying resonance frequency and the excitation pulse frequency leads to a ringing behavior $[7,8]$, and induces temporal distortions of the pulse [9].

In order to maintain the benefit of light localization throughout the pulse excitation and to control the intracavity field dynamics, we study in this article the behavior of a nonlinear microcavity excited by a tailored chirped pulse. The spectral components of the excited pulse are set to compensate for the nonlinear frequency drift of the cavity resonance. This ap- proach is based on the pulse shaping techniques that are commonly used for the coherent excitation of atomic and molecular systems [10]-[12], and which start now to be theoretically investigated in nonlinear microcavities [13, 14]. Sandhu et. al. [13] have studied a phase-shaped pulse excitation that compensates for the intrinsic linear dispersion of the cavity resonance, in order to reduce the switching threshold power of a bistable Kerr microcavity. Very recently, Kristensen et. al. [14] have demonstrated fast switching of a pure-Kerr microcavity in a controlled two-pulse excitation scheme.

While references [13] and [14] deal with cavities operating under pure Kerr effect, we consider a more realistic nonlinear semiconductor microcavity driven by the optical Kerr effect, the two-photon absorption (TPA), the free carrier refraction (FCR) and absorption (FCA) effects induced by the free carriers generated by TPA. In a first section, we present the model used to simulate the dynamic behavior of the intracavity field. It is based on the coupled mode theory [15, 16] and includes the nonlinear effects listed above. We next study the dynamics of a silicon based microcavity under a linearly chirped pulse excitation. By compensating for the nonlinear frequency drift of the cavity resonance, using an appropriate phase relation between the spectral components of the input pulse, we show an improvement in the intracavity timeaveraged stored energy and an enhancement of the nonlinear interactions. We conclude by discussing the interest of such a coherent excitation as a means to control the dynamics of nonlinear microcavities. 


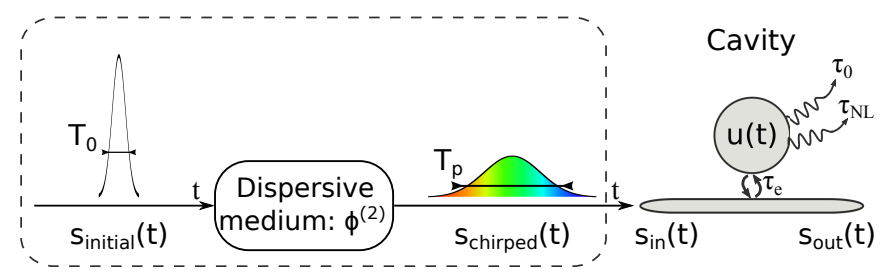

FIG. 1 Side coupled cavity geometry excited by an input pulse $s_{\text {in }}(t)$. Left inset: scheme used to linearly chirped an initial Fourier-transform limited pulse $s_{\text {initial }}(t)$.

\section{MODEL}

We consider the nonlinear dynamics of an electromagnetic field circulating inside a cavity coupled to a bus waveguide in a side-coupled geometry depicted in Figure 1. The field amplitude is defined as $u(t)=\sqrt{P_{u}(t) \tau_{R}} \mathrm{e}^{i \phi_{u}(t)}$, where $P_{u}(t)$ is the intra-cavity optical power, $\phi_{u}(t)=\arg [u(t)]$ the intra-cavity field phase, $\tau_{R}$ the cavity round-trip time. Using the coupled mode theory, its temporal evolution is given by $[15,16]$

$$
\begin{aligned}
\frac{\mathrm{d} u}{\mathrm{~d} t}= & -\left(\frac{1}{\tau}+\frac{1}{\tau_{\mathrm{NL}}(t)}\right) u(t)+i \omega_{\mathrm{res} 0}\left(1+\frac{\Delta \omega_{\mathrm{res}}(t)}{\omega_{\mathrm{res} 0}}\right) u(t) \\
& +\sqrt{\frac{2}{\tau_{e}}} s_{\mathrm{in}}(t)
\end{aligned}
$$

where $\left|s_{\text {in }}(t)\right|^{2}$ is the instantaneous power of the input signal and $\omega_{\text {res } 0}$ is the resonance frequency of the cavity set in the linear regime. The linear cavity photon lifetime $\tau / 2$ corresponds to $1 / \tau=1 / \tau_{0}+1 / \tau_{e}$, where $\tau_{0} / 2$ and $\tau_{e} / 2$ are the photon lifetimes respectively related to the intrinsic cavity linear losses and to the coupling constant between the waveguide and the cavity. The nonlinear effects are included in the first two terms of the right-hand side of Eq. (1). The parameter $\tau_{\mathrm{NL}}$, which depends on the intracavity mode amplitude $u$, depicts the modification of the cavity photon lifetime through the nonlinear absorption effects. The time dependent frequency shift of the cavity resonance induced by the nonlinear refractive index change of the material is described by the term $\Delta \omega_{\text {res }}(t)$. Notice that Eq. (1) assumes a slowly-varying field amplitude $u(t)$ within the cavity round-trip time $\tau_{R}$.

In the next section, we will consider the nonlinear behavior of a silicon based microcavity operating around $1550 \mathrm{~nm}$. Although one photon energy $(0.8 \mathrm{eV})$ is smaller than the silicon band gap energy $(\simeq 1.1 \mathrm{eV})$, nonlinear absorption occurs due to two photon absorption, which is accompanied by the simultaneous generation of free carriers. The two related nonlinear absorption effects, respectively TPA and FCA, are included in the parameter $\tau_{\mathrm{NL}}(t)$ through the relation $1 / \tau_{\mathrm{NL}}(t)=1 / \tau_{\mathrm{TPA}}(t)+1 / \tau_{\mathrm{FCA}}(t)[16]$, with

$$
\frac{1}{\tau_{\mathrm{TPA}}(t)}=\frac{\beta_{\mathrm{TPA}} c^{2}}{2 n_{0}^{2} V}|u(t)|^{2}, \quad \frac{1}{\tau_{\mathrm{FCA}}(t)}=\frac{\sigma_{a} c}{2 n_{0}} N(t) .
$$

The TPA photon lifetime $\tau_{\text {TPA }} / 2$ depends on the two-photon absorption coefficient $\beta_{\mathrm{TPA}}$, the effective linear refractive index of the cavity material $n_{0}$, the speed of light $c$, and the Kerr volume $V$. The FCA photon lifetime $\tau_{\mathrm{FCA}} / 2$ is expressed in terms of the FCA cross section $\sigma_{a}$ and the free carrier density
$N(t)$ with a temporal evolution governed by:

$$
\frac{\mathrm{d} N(t)}{\mathrm{d} t}+\frac{1}{\tau_{c}} N(t)=\frac{\beta_{\mathrm{TPA}}}{2 \hbar \omega}\left(\frac{c|u(t)|^{2}}{n_{0} V}\right)^{2},
$$

where $\tau_{c}$ is the carrier lifetime. For the sake of simplicity, we have approximated the free-carrier effects volume to the Kerr volume. We have also neglected the linear dispersion of the cavity material such as the group index is approximated to $n_{0}$. The nonlinear refractive index variation $\Delta n(t) / n_{0}$, governed by both the Kerr effect and the FCR, modifies the resonance frequency through the relation:

$$
\frac{\Delta \omega_{\mathrm{res}}(t)}{\omega_{\mathrm{res} 0}}=-\frac{\Delta n(t)}{n_{0}}=-\frac{n_{2} c}{n_{0}^{2} V}|u(t)|^{2}-\frac{\sigma_{r}}{n_{0}} N(t) .
$$

The first term on the right-hand side corresponds to the optical Kerr effect, where $n_{2}$ is the Kerr coefficient, and the second one is related to the FCR effect, with $\sigma_{r}$ the effective volume of free carrier refraction. According to the analysis conducted in [17], and considering our pulse excitation condition (few ps pulse duration and low repetition time around $10 \mathrm{~ns}$ ), we have neglected the thermal refractive index variation related to the fast dynamics of the free carriers. Indeed, it has been evaluated two orders of magnitude lower than the free carrier refractive index change. Using Eqs. (1)-(4), we next study the temporal evolution of the intra-cavity field intensity.

\section{NONLINEAR MICROCAVITY DYNAMICS}

We consider a silicon based microcavity with a length $L=900 \mathrm{~nm}$ and an effective mode area $S_{\text {eff }}=300 \times 300 \mathrm{~nm}^{2}$, which leads to a cavity mode volume $V=0.081 \mu \mathrm{m}^{3}$. The intrinsic and extrinsic losses are set to $\tau_{0}=49.4 \mathrm{ps}$ and $\tau_{e}=15 \mathrm{ps}$, leading to a quality factor $Q=\omega \tau / 2=7,000$ (with $\tau=11.5 \mathrm{ps}$ ). The nonlinear parameters for silicon around $1550 \mathrm{~nm}$ [16] are: $n_{2}=4.4 \times 10^{-18} \mathrm{~m}^{2} / \mathrm{W}$, $\beta_{\mathrm{TPA}}=8.4 \times 10^{-12} \mathrm{~m} / \mathrm{W}, \quad \sigma_{r}=-1.34 \times 10^{-27} \mathrm{~m}^{3}$, and $\sigma_{a}=14.5 \times 10^{-22} \mathrm{~m}^{2}$. As the effective linear refractive index depends on the cavity structure, which is not defined here, it is set to the linear refractive index of silicon $n_{0}=3.48$, leading to $\tau_{R}=n_{0} L / c=10 \mathrm{fs}$. Moreover, the carrier lifetime $\tau_{c}$ is assumed to be $0.4 \mathrm{~ns}$ (as measured in ref [16]), which is much longer than both the pulse duration and the cavity photon lifetime. We assume here that $\tau_{e}$ is not affected by the nonlinear effects.

In this section, we study an excitation under a Fouriertransform-limited gaussian pulse with a full width at $1 / e$ in intensity of $3.4 \tau$ and an incident peak power of $23 \mathrm{~mW}$, corresponding to an energy of $780 \mathrm{fJ}$. The pulse duration is arbitrary chosen such that it is longer than $\tau$ in order to avoid a transient excitation of the cavity [18]. When the pulse carrier frequency coincides with the resonance frequency $\omega_{\text {res} 0}$, the temporal evolution of the intra-cavity power $P_{u}(t)$ is represented by the black solid line in Figure 2(a). The comparison with the incident pulse profile, given by the dashed line, shows a sudden drop of the intra-cavity power at the time $t=-0.5 \tau$ followed by a damped oscillation. It actually coincides with the strong detuning of the cavity resonance $\Delta \omega_{\text {res }}(t)$ reported in Figure 2(b) with the black 

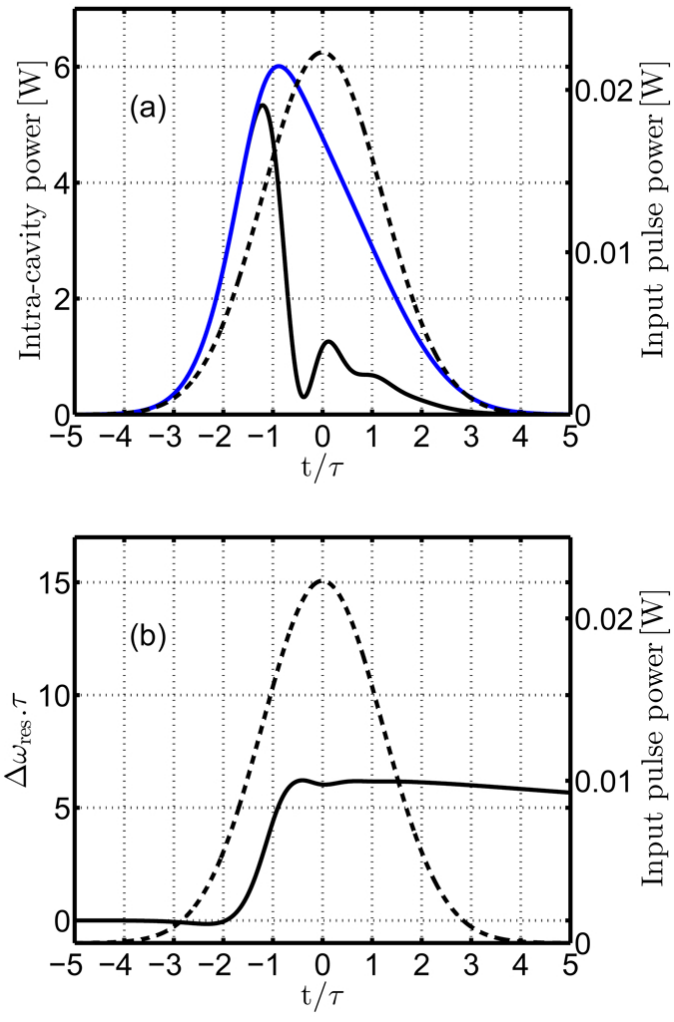

FIG. 2 (a) Temporal evolution and (b) resonance frequency drift of the intra-cavity power under an on-resonance gaussian excitation with (black curves) and without (blue curve) considering the nonlinear refractive effects. The input pulse profile is plotted with a dashed line.

curve, which shows a frequency blue-shift of about $6 / \tau$ (i.e. 3 times the spectral linewidth of the cavity resonance). The cavity detuning is mainly driven by the refractive index variation induced by the free carriers generated by TPA, which dominates the optical Kerr effect. The sign of the frequency shift is in accordance with the negative value of $\sigma_{r}$.

A physical insight of the damped oscillation of the intra-cavity power can be given by rewriting Eq. (1) to derive the temporal evolution of the energy term $|u(t)|^{2}$ of the intra-cavity resonant mode

$$
\begin{aligned}
& \frac{\mathrm{d}|u|^{2}}{\mathrm{~d} t}+2\left(\frac{1}{\tau}+\frac{1}{\tau_{\mathrm{NL}}(t)}\right)|u(t)|^{2} \\
= & 2 \sqrt{\frac{2}{\tau_{e}}}|u(t)|\left|s_{\text {in }}(t)\right| \cos \left[\phi_{u}(t)-\phi_{\text {in }}(t)\right],
\end{aligned}
$$

where $\phi_{u}(t)=\arg [u(t)]$ is the self-phase modulation term of the intra-cavity field, and $\phi_{i n}(t)=\arg \left[s_{\text {in }}(t)\right]$ is the input pulse phase. The time varying phase $\phi_{u}(t)$ results from the nonlinear refractive index change, while $\phi_{\text {in }}$ is constant in the present case. This equation describes the damping (lefthand side) of a source term (right-hand side), namely the beating between the intra-cavity field and the input pulse. The mismatch between the time varying phase $\phi_{u}(t)$ of the intracavity field and the fixed phase of the input pulse gives rise to the oscillating behavior with a damping rate governed by both the linear and nonlinear losses. Consequently, this phase mismatch tends to reduce the intra-cavity field energy, which could be counter-balanced by a proper tailoring of the input pulse phase $\phi_{i n}(t)$ as it will be shown in Section 4.
In order to show that the reduction of the stored energy is mainly governed by the nonlinear refractive effects, we next perform a simulation for which the nonlinear frequency shift is omitted, i.e. by setting $\Delta \omega_{\text {res }}(t)=0$ in Eq. 1 . Note that such a situation would be equivalent to consider an input pulse with a tailored phase-shape $\phi_{i n}(t)=\phi_{u}(t)$, perfectly compensating the nonlinear refractive induced frequency drift of the cavity resonance. The corresponding temporal evolution of the intra-cavity field is represented by the solid blue line in Figure 2(a). Despite nonlinear absorption effects, the stored energy is higher than in the former simulation that includes the refractive effects (curves in black solid line). To quantify this improvement, we introduce a magnification factor defined as the ratio between the time averaged intra-cavity field power and the time-averaged input pulse power:

$$
\mathcal{M}_{\text {eff }}=\frac{\int_{-\infty}^{+\infty} P_{u}(t) \mathrm{d} t}{\int_{-\infty}^{+\infty}\left|s_{\mathrm{in}}(t)\right|^{2} \mathrm{~d} t}
$$

According to this definition, the calculated magnification factor for the black curve in Figure 2(a) is $\mathcal{M}_{\text {eff }}=130$, while it reaches $\mathcal{M}_{\text {eff }}=265$ for the blue curve. Moreover, the generated carrier density calculated for the latter is 3 times higher than in the former, which shows an enhancement of the lightmatter interaction within the cavity in the case of a tailored phase-shaped pulse.

In the next section, we will demonstrate that, using an appropriate linearly chirped pulse, the beating effect between the incident and the intra-cavity fields is reduced and its interaction with the intra-cavity nonlinear material can be enhanced.

\section{COHERENT EXCITATION OF A NONLINEAR MICROCAVITY}

In the case of a gaussian pulse and assuming a carrier lifetime longer than the pulse duration, the resonance drift depicted in Figure 2(b) can be approximated by an error function (see Eq. (3) and [19]). Therefore, we can study the cavity behavior under a linearly chirped pulse excitation with a spectral phase relation that fairly approximates the resonance drift. A linearly chirped pulse is achieved through the propagation of a transform limited pulse $s_{\text {initial }}$ inside a second order dispersive medium (see left-inset in Figure 1).

Hereafter, the initial transform-limited pulse $s_{\text {initial }}$ follows a gaussian shape :

$$
s_{\text {initial }}(t)=\sqrt{P_{0}} \exp \left[\frac{-t^{2}}{2 T_{0}^{2}}\right] \mathrm{e}^{i\left(\omega_{\text {res } 0}+\delta \omega\right) t},
$$

where $\delta \omega=\omega_{0}-\omega_{\text {res } 0}$ is the frequency detuning between the pulse and the cavity resonance. At the output of the dispersive medium, the chirped pulse is expressed

$$
s_{\text {chirped }}(t)=\sqrt{\frac{T_{0}}{T_{p}} P_{0}} \exp \left[-\frac{t^{2}}{2 T_{p}^{2}}+i \frac{1}{2} \alpha t^{2}\right] \mathrm{e}^{i\left(\omega_{\text {res } 0}+\delta \omega\right) t},
$$

where $T_{p}$ and $\alpha$ are related to the second order dispersion co- 


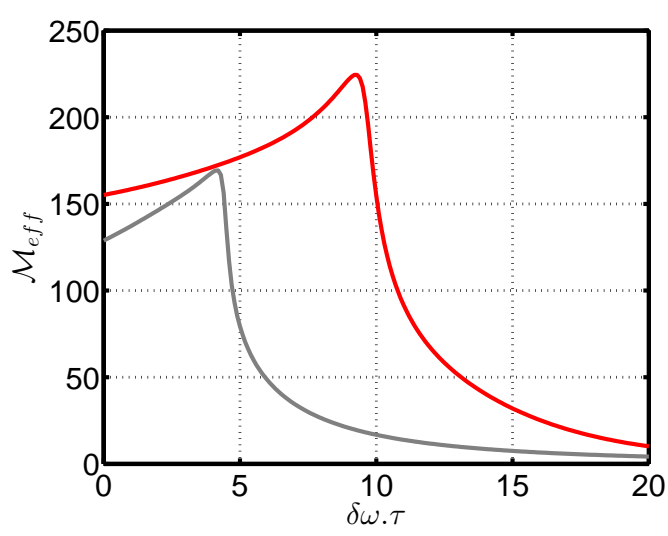

FIC. 3 Magnification factor variation with the frequency detuning $\delta \omega$ for both the chirped pulse (red line) and the transform-limited pulse (grey line).

$$
\begin{aligned}
\text { efficient } \phi^{(2)} & =\left.\frac{\mathrm{d}^{2} \phi}{\mathrm{d} \omega^{2}}\right|_{\omega_{0}}: \\
T_{p} & =T_{0} \sqrt{1+\left(\frac{\phi^{(2)}}{T_{0}^{2}}\right)^{2}}, \quad \alpha=\frac{\phi^{(2)}}{T_{0}^{4}+\phi^{(2)^{2}}} .
\end{aligned}
$$

For a strongly chirped pulse, we get $T_{p} \approx\left|\phi^{(2)}\right| / T_{0}$ and $\alpha \approx 1 / \phi^{(2)}$.

We first need to set the parameters $T_{0}, P_{0}, \delta \omega$ of the initial pulse and the dispersion coefficient $\phi^{(2)}$. For the sake of comparison, the envelope characteristic of the chirped pulse is kept equal to that of the Fourier-transform limited gaussian pulse used in the previous section. Thus, we set its peak power to $23 \mathrm{~mW}$, the pulse duration to $T_{p}=1.7 \tau$, which gives a pulse energy of $780 \mathrm{fJ}$. The spectral linewidth of the pulse should now be consistent with the frequency shift experienced by the cavity resonance. As we have anticipated that 3 times more carrier density is expected with a tailored phase-shaped pulse, i.e. $\phi_{\text {in }}(t)=\phi_{u}(t)$, the cavity blue-shift should reach $18 / \tau$ (3 times the $6 / \tau$ blue-shift reported in Figure 2(b)). Following this expectation, the spectral linewidth of $s_{\text {initial }}(t)$ is set to $20 / \tau$, corresponding to a pulse duration $T_{0}=\tau / 10$. The dispersion coefficient calculated from Eq. 9 is then set to $\phi^{(2)}=0.17 \tau^{2}=22 \mathrm{ps}^{2}$. By neglecting the propagation loss through the dispersive medium, the peak power of the initial pulse should be equal to $P_{0}=381 \mathrm{~mW}$ (see Eq. 8).

The remaining parameter is the initial detuning frequency $\delta \omega$. Intuitively, the chirped-pulse should be blue-shifted respectively to the initial cavity resonance by one-half of the nonlinear frequency blue-shift. A more accurate analysis is conducted by computing the intra-cavity field dynamics, setting $s_{\text {in }}(t)=s_{\text {chirped }}(t)$ in Eq. (1) with the previously defined parameters, for $\delta \omega$ detunings varying between 0 and $20 / \tau$. Following the previous section, we have plotted in Figure 3 the effective magnification factor $\mathcal{M}_{\text {eff }}$ calculated for the different detuning values (see the red curve). The time-averaged stored energy increases along with a blue detuning. The maximum value $\mathcal{M}_{\text {eff }}=225$ is obtained for $\delta \omega=9 / \tau$, which is nearby the value we have anticipated. For a larger detuning, the initial coupling efficiency of the pulse with the cavity resonance is too weak to efficiently generate free carriers and to initiate a blue shift of the cavity.
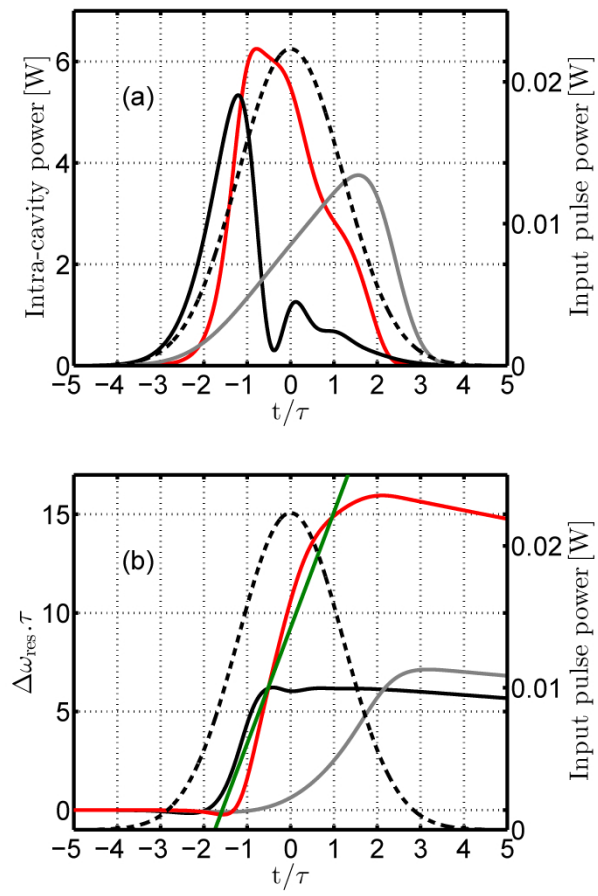

FIG. 4 (a) Intra-cavity power and (b) cavity resonance frequency drift in the case of an initially on resonance transform-limited pulse (black lines), a transform-limited pulse with a numerically optimized frequency detuning (grey lines) and a linearly chirped pulse (red line) whose chirp is plotted in green (b). The input pulse profile is plotted with dashed line.

The temporal evolution of the intra-cavity power achieved with the previously optimized chirped-pulse excitation is plotted with red solid line in Figure 4(a). Its temporal evolution is compared to the envelop intensity of the excited pulse (black dashed line) and to the intra-cavity power (black solid line) calculated in the previous section, with $\delta \omega=0$. The dynamics of the resonance frequency drift achieved in both cases are reported in Figure 4(b). In the case of the tailored chirped pulse excitation, the resonance frequency drift reaches $16 / \tau$, which is close to the expected value of $18 / \tau$ in the case of the perfect phase control. The green line in Figure 4(b) shows the linear chirp of the input pulse, whose slope is $\alpha=1 / \phi^{(2)}$, which tangentially fits the resonance frequency drift during the whole excitation.

As the Fourier-transform-limited gaussian pulse exhibits a ringing behavior on the intra-cavity field dynamics, the intracavity field shape under the chirped pulse excitation undergoes less distortions and leads to a higher magnification factor. This improvement is due to an accurately tailored chirped pulse, whose instantaneous frequency coincides with the nonlinear resonance drift, which allows the light to efficiently build-up inside the cavity.

Lastly, we consider the case of the Fourier-transform-limited gaussian pulse with a non-zero detuning in order to emphasize the importance of the pulse phase-shaping. Similarly to the chirped pulse excitation, the intra-cavity field dynamics is computed for various values of $\delta \omega$. The calculated effective magnification factors are reported with the grey curve in Figure 3. The maximum value $\mathcal{M}_{\text {eff }}=170$ is reached for $\delta \omega \approx 4 / \tau$. The corresponding intra-cavity dynamics plotted 
in Figure 4(a) (grey line) does not exhibit the ringing behavior that can be seen for the $\delta \omega=0$ non-chirped gaussian pulse. Although this initial detuning slightly improves $\mathcal{M}_{\text {eff }}$, it does not significantly increase the carrier density as the frequency blue-shift of the cavity resonance remains about $7 / \tau$ (grey curve in Figure 4(b)).

\section{CONCLUSION}

The nonlinear dynamics of an optical microcavity subject to a nonlinear refractive induced frequency shift has been discussed in this article. Although an optical microcavity is used to enhance the light matter interaction, we have emphasized that the benefit of the light localization effect cannot be maintained throughout the pulse excitation because of the nonlinear refractive index change.

As an illustration, we have theoretically studied the intracavity intensity dynamics for a silicon based microcavity under a coherent pulse excitation. We have shown that a tailored linearly chirped pulse, for which the instantaneous frequency coincides with the nonlinear resonance drift, ensures a buildup of the intra-cavity energy. By doing so the light localization effect during the pulse excitation can be maintained, which leads to an enhancement of the intra-cavity nonlinear interaction. In our example, we have compared the excitation between a Fourier-transform-limited gaussian pulse and a tailored chirped pulse excitation. While the two pulses share the same gaussian shape with equal energy (780 fJ) and pulse duration (6.8 times the cavity photon lifetime), an enhancement by a factor 3 of the free carrier density generated by twophoton absorption is reported in the latter case. Moreover, the intra-cavity field amplitude dynamics undergoes less deformation, illustrating the control of nonlinear dynamics by means of the pulse phase-shaping.

Besides the control of the nonlinear dynamics, such a coherent excitation enables to manipulate the effective optical bandwidth of microcavities. Indeed, we have performed an efficient transfer of energy between an intra-cavity medium and a pulse whose spectral linewitdth is 10 times larger than that of the linear cavity. Experiments are presently under progress to verify our theoretical predictions and to study the dependence between the chirp value and the energy of the input pulse.

The coherent excitation could be an efficient meaning to improve and to dynamically control the operation of microcavity-based all-optical devices. It could address the issue of their energy consumption and the control of more complex structures, such as coupled microcavities.

\section{Acknowledgements}

The authors acknowledge Robert Frey and Gilles Pauliat for highly valuable comments and discussions.

\section{References}

[1] M. Soljačić, and J. D. Joannopoulos, "Enhancement of nonlinear effects using photonic crystals," Nat. Mater. 3, 211 (2004).

[2] T. Tanabe, M. Notomi, S. Mitsugi, A. Shinya, and E. Kuramochi, “Alloptical switches on a silicon chip realized using photonic crystal nanocavities," App. Phys. Lett. 87, 151112 (2005).

[3] F. Raineri, C. Cojocaru, P. Monnier, A. Levenson, R. Raj, C. Seassal, $X$. Letartre, and P. Viktorovitch, "Ultrafast dynamics of the thirdorder nonlinear response in a two-dimensional InP-based photonic crystal," App. Phys. Lett. 85, 1880 (2004).

[4] C. Husko, A. De Rossi, S. Combrié, Q. V. Tran, F. Raineri, and C. W Wong, "Ultrafast all-optical modulation in GaAs photonic crystal cavities," App. Phys. Lett. 94, 021111 (2009).

[5] K. Nozaki, T. Tanabe, A. Shinya, S. Matsuo, T. Sato, H. Taniyama, and M. Notomi, "Sub-femtojoule all-optical switching using a photonic-crystal nanocavity," Nat. Photon. 4, 477 (2010).

[6] V. Eckhouse, I. Cestier, G. Eisenstein, S. Combrié, G. Lehoucq, and A. De Rossi, "Kerr-induced all-optical switching in a GalnP photonic crystal Fabry-Perot resonator" Opt. Express 20, 8524 (2012).

[7] Z. K. Ioannidis, P. M. Radmore, and I. P. Giles, "Dynamic response of an all-fiber ring resonator," Opt. Lett. 13, 422 (1988).

[8] Y. Dumeige, S. Trebaol, L. Ghişa, T. K. N. Nguyên, H. Tavernier, and $P$. Féron, "Determination of coupling regime of high- $Q$ resonators and optical gain of highly selective amplifiers," J. Opt. Soc. Am. B 25, 2073 (2008).

[9] D. Vujic, and S. John, "Pulse reshaping in photonic crystal waveguides and microcavities with Kerr nonlinearity: Critical issues for all-optical switching," Phys. Rev. A 72, 013807 (2005).

[10] A. M. Weiner, "Femtosecond pulse shaping using spatial light modulators," Rev. Sci. Instrum. 71, 1929 (2000).

[11] S. Zamith, J. Degert, S. Stock, B. de Beauvoir, V. Blanchet, M. A. Bouchene, and B. Girard, "Observation of Coherent Transients in Ultrashort Chirped Excitation of an Undamped Two-Level System," Phys. Rev. Lett. 87, 033001 (2001).

[12] D. Meshulach, and Y. Silberberg, "Coherent quantum control of two-photon transitions by a femtosecond laser pulse," Nature 396, 239 (1998).

[13] S. Sandhu, M. Povinelli, and S. Fan, "Enhancing optical switching with coherent control," App. Phys. Lett. 96, 231108 (2010).

[14] P. T. Kristensen, M. Heuck, and J. Mork, "Optimal switching using coherent control," App. Phys. Lett. 102, 041107 (2013).

[15] H. A. Haus, Waves and fields in optoelectronics (Prentice-Hall, New Jersey, 1984).

[16] P. Barclay, K. Srinivasan, and 0. Painter, "Nonlinear response of silicon photonic crystal microresonators excited via an integrated waveguide and fiber taper," Opt. Express 13, 801 (2005).

[17] D. Prelewitz, and T. Brown, "Optical limiting and free-carrier dynamics in a periodic semiconductor waveguide," J. Opt. Soc. Am. B 11, 304-312 (1994).

[18] M. Heuck, P.T. Kristensen, and J. Mørk, "Energy-bandwidth tradeoff in all-optical photonic crystal microcavity switches," Opt. Express 19, 18410 (2011).

[19] A. Baron, N. Dubreuil, P. Delaye, R. Frey, and G. P. Agrawal, "Raman amplification of optical pulses in silicon nanowaveguides: Impact of spectral broadening of pump pulses," J. Europ. Opt. Soc. Rap. Public. 6, 11030 (2011). 